\title{
Reliability Characterization of Binary-Imaged Multi-State Coherent Threshold Systems
}

\author{
Ali Muhammad Ali Rushdi \\ Department of Electrical and Computer Engineering, \\ Faculty of Engineering, King Abdulaziz University, \\ P. O. Box 80204, Jeddah, 21589, Saudi Arabia. \\ Corresponding author: arushdi@kau.edu.sa \\ Fares Ahmad Muhammad Ghaleb \\ Department of Electrical and Computer Engineering, \\ Faculty of Engineering, King Abdulaziz University, \\ P. O. Box 80204, Jeddah, 21589, Saudi Arabia. \\ E-mail: fares.ghaleb@yahoo.com
}

(Received December 7, 2019; Accepted March 26, 2020)

\begin{abstract}
A notable reliability model is the binary threshold system (also called the weighted- $k$-out-of- $n$ system), which is a dichotomous system that is successful if and only if the weighted sum of its component successes exceeds or equals a particular threshold. The aim of this paper is to extend the utility of this model to the reliability analysis of a homogeneous binary-imaged multi-state coherent threshold system of $(m+1)$ states, which is a non-repairable system with independent non-identical components. The paper characterizes such a system via switching-algebraic expressions of either system success or system failure at each non-zero level. These expressions are given either (a) as minimal sum-of-products formulas, or (b) as probability-ready expressions, which can be immediately converted, on a one-toone basis, into probabilities or expected values. The various algebraic characterizations can be supplemented by a multitude of map representations, including a single multi-value Karnaugh map (MVKM) (giving a superfluous representation of the system structure function $S),(m+1)$ maps of binary entries and multi-valued inputs representing the binary instances of $S$, or $m$ maps, again of binary entries and multi-valued inputs, but now representing the success/failure at every non-zero level of the system. We demonstrate how to reduce these latter maps to conventional Karnaugh maps (CKMs) of much smaller sizes. Various characterizations are inter-related, and also related to pertinent concepts such as shellability of threshold systems, and also to characterizations via minimal upper vectors or via maximal lower vectors.
\end{abstract}

Keywords- System reliability, Probability-ready expression, Threshold system, Multi-state system, Multi-valued Karnaugh map, Minimal upper vector, Maximal lower vector.

\section{Introduction}

Two of the most fruitful and successful paradigms of system reliability modeling are those of the multi-state model (Barlow and Wu, 1978; Boedigheimer and Kapur, 1994; El-Neweihi et al., 1978; Griffith, 1980; Hudson and Kapur, 1983; Janan, 1985; Kumar and Singh, 2018; Lisnianski and Levitin, 2003; Mo et al., 2015; Ram, 2013; Tian et al., 2008; Wood, 1985), and the threshold model (Rushdi, 1990; Rushdi and Alturki, 2015, 2018; Rushdi and Bjaili, 2016), also known as the weighted $k$-out-of- $n$ model (Eryilmaz, 2015; Li et al., 2016; Eryilmaz and Bozbulut, 2019; Salehi et al., 2019). Few attempts have been made to combine the two models (Ding et al., 2012; Khorshidi et al., 2015; Meenkashi et al., 2019). This paper is a serious attempt to unify the two models for the case of a coherent system with a well-defined binary image (Ansell and Bendell, 1987). This model of a multi-state coherent threshold system is very versatile, indeed. It inherits 
International Journal of Mathematical, Engineering and Management Sciences

Vol. 6, No. 1, 309-321, 2021

https://doi.org/10.33889/IJMEMS.2021.6.1.020

all strengths of the multi-state model and the threshold model, and might prove to possess applications that are far wider than those of its constituent sub-models. This paper explores and inter-relates a variety of algebraic, map, and vector characterizations of this model, and explains how to evaluate its reliability via switching-algebraic techniques and tools used in the binary case (Rushdi and Goda, 1985; Rushdi and Abdulghani, 1993; Rushdi and Hassan, 2015, 2016).

The organization of the remainder of this paper is as follows. Section 2 offers a mathematical description of a binary-imaged multi-state coherent threshold system, while Sec. 3 specifies a special case of such a system that serves as a running example for this paper. Section 4 presents four switching-algebraic characterizations of the example system via minimal sum-of-products formulas and probability-ready expressions (PREs) of the success and failure at each non-zero system level. Section 5 presents an exhaustive (and superfluous) listing of system states via a multi-valued Karnaugh map (MVKM) (Rushdi, 2018), and then reduces this large-size map to three much smaller binary maps, and finally adds two other characterizations in terms of minimal upper vectors and maximal lower vectors. Section 6 obtains expected-value or reliability expressions via direct transformations of the PRE switching expressions. Section 7 concludes the paper.

\section{Description of a Binary-Imaged Multi-State Coherent Threshold System}

In this Section, we employ terminology and notation used recently by Rushdi and Al-Amoudi (2018), Rushdi and Alsayegh (2019) and Rushdi (2019). A multi-state system is specified by its structure or success function $S(\boldsymbol{X})$, which is given as

$$
S:\left\{0,1, \cdots, m_{1}\right\} \times\left\{0,1, \cdots, m_{2}\right\} \times \ldots \times\left\{0,1, \cdots, m_{n}\right\} \rightarrow\{0,1, \cdots, M\} .
$$

When $m_{1}, m_{2}, \cdots, m_{n}$ and $M$ have a common value $m$, the system is said to be homogeneous, and when this common $m$ equals 2 , the system reduces to a two-state (binary) system. The main thesis of this paper is that a homogeneous binary-imaged multi-state system (such as a binaryimaged threshold one) is equivalent to $m$ binary systems, and hence a multi-valued analysis of this system might be replaced by $m$ switching-algebraic analyses of binary systems. In fact, we can replace the multi-valued function $S$ which has values belonging to $\{0,1, \cdots, m\}$ by its set of $(m+1)$ binary instances $S\{j\}$, which $\in\{0,1\}$ such that

$$
S\{j\}=\{S(\boldsymbol{X})=j\}, \quad 0 \leq j \leq m .
$$

The instances $S\{j\}$ form an orthonormal set, since they are exhaustive $\left(\mathrm{V}_{j=0}^{m} S\{j\}=1\right)$ and mutually disjoint $\left(S\left\{j_{1}\right\} S\left\{j_{2}\right\}=0\right.$ for $\left.j_{1} \neq j_{2}\right)$. Likewise, we replace each of the multi-valued input variables $X_{i}$ by its set of binary instances $\left\{X_{i}\{j\}, 0 \leq j \leq m\right\}$ (Rushdi, 2019). Since we deal herein with a binary-imaged system, we restrict our attention to what we call the system success at the non-zero level $j(1 \leq j \leq m)$, defined by

$$
S\{\geq j\}=S\{j, j+1, \cdots, m\}=S\{j\} \vee S\{j+1\} \vee \cdots \vee S\{m\},
$$

or its complement, the system failure at level $j$

$$
S\{<j\}=S\{\leq(j-1)\}=S\{0,1, \cdots, j-1\}=S\{0\} \vee S\{1\} \vee \cdots \vee S\{j-1\},
$$


International Journal of Mathematical, Engineering and Management Sciences

Vol. 6, No. 1, 309-321, 2021

https://doi.org/10.33889/IJMEMS.2021.6.1.020

and confine the analysis of the multi-state system to an exploration of $S\{\geq j\}(1 \leq j \leq m)$ as a function of $\boldsymbol{X}\{\geq j\}$ solely (or of $S\{\leq j\}(0 \leq j \leq(m-1))$ as a function of $\boldsymbol{X}\{\leq j\}$ solely). Since the system is binary-imaged, it is a dominant multi-state one (Zuo et al., 2003; Huang and Zuo, 2004).

We now introduce the concept of a binary-imaged multi-state threshold system as one that acts as a binary threshold system at each of its $m$ non-zero levels, namely

$$
\{S\{\geq j\}=1\} \Leftrightarrow\left\{\boldsymbol{W}^{T}(j) \boldsymbol{X}\{\geq j\} \equiv \sum_{i=1}^{n} W_{i}(j) X_{i}\{\geq j\} \geq T(j)\right\},
$$

where $W_{i}(j)$ is the weight of element $i$ at level $j$, and $T(j)$ is the threshold at level $j$. Properties of such a system are expected to replicate those of a binary threshold system, studied extensively by Rushdi (1990), Rushdi and Alturki $(2015,2018)$ and Rushdi and Bjaili (2016).

We restrict ourselves herein to a coherent multi-state system, and hence our system must satisfy certain aspects of monotonicity, causality and relevancy (Rushdi et al., 2016; Rushdi and Rushdi, 2017, 2018). In particular, the binary systems at the non-zero levels are coherent, so that each of the weights and threshold of each of them is strictly positive (Rushdi, 1990).

\section{Description of a Particular Running Example}

In this section, we specialize our description of a binary-imaged multi-state coherent system to a specific coherent example, in which the number of components is $n=3$, the number of non-zero levels is $m=3$, the various non-zero levels share the same weights (which are different for different components)

$W_{1}(j)=8, W_{2}(j)=4, W_{3}(j)=2, W_{4}(j)=1$,

such that the threshold at level $j$ is

$T(j)=2 j+1$.

Specifically, $T(1)=3, T(2)=5$, and $T(3)=7$. We stress that this system has the same weight vector at each non-zero level, but it has increasing thresholds. It combines several threshold systems of practical significance and importance (Rushdi and Alturki, 2015). Had the system weights in (6) been equal, the system would have reduced to the special case of a generalized $k$ out-of- $n$ : $G$ system with increasing $k$, a binary-imaged (and hence, dominant) multi-state system (Zuo et al., 2003; Huang and Zuo, 2004).

\section{Algebraic Characterizations}

Table 1 lists four sets of three equations each, wherein each individual set is a complete characterization of the system under study. The top-left set of equations (equations (8)-(10)) in Table 1 give each $S\{\geq j\}$ as a function of $\boldsymbol{X}\{\geq j\}$ (for $j=3,2$ and 1), which depicts system success at level $j$ (upper states) in a minimal sum-of-products form. The products involved are prime implicants (PIs) of the respective functions. Equations (8)-(10) are directly deduced from the system definition in (5)-(7) (see e.g., Rushdi and Alturki, 2015). The top-right set of equations (equations (11)-(13)) are obtained by complementation and application of De Morgan's rules to the former equations (8)-(10). These latter equations express each $S\{\leq j\}$ as a function of $\boldsymbol{X}\{\leq j\}$ 
International Journal of Mathematical, Engineering and Management Sciences

Vol. 6, No. 1, 309-321, 2021

https://doi.org/10.33889/IJMEMS.2021.6.1.020

(for $j=2,1$, and 0 ), which depicts the system failure at level $(j+1)$ (lower states for level $(j+$ 1)).

Table 1. A listing of minimal and PRE expressions of system success and failure at each non-zero level $j(j=1,2,3)$. Crossing the vertical axis of the table from left to right involves complementation, while crossing the horizontal axis of the table from top to bottom involves orthogonalization (disjointing of terms).

\begin{tabular}{|c|c|c|}
\hline & Upper (Success) Expressions & Lower (Failure) Expression \\
\hline \multirow{3}{*}{$\underset{1}{\operatorname{Minima}}$} & $\begin{array}{l}S\{\geq 3\} \\
=X_{1}\{\geq 3\} \\
\vee X_{2}\{\geq 3\} X_{3}\{\geq 3\} X_{4}\{\geq 3\}\end{array}$ & $\begin{array}{c}S\{\leq 2\}=X_{1}\{\leq 2\}\left(X_{2}\{\leq 2\} \vee X_{3}\{\leq 2\}\right. \\
\left.\vee X_{4}\{\leq 2\}\right)\end{array}$ \\
\hline & $\begin{array}{l}S\{\geq 2\} \\
=X_{1}\{\geq 2\} \vee X_{2}\{\geq 2\}\left(X_{3}\{\geq 2\}\right. \\
\left.\vee X_{4}\{\geq 2\}\right)\end{array}$ & $\begin{array}{l}S\{\leq 1\} \\
=X_{1}\{\geq 1\}\left(X_{2}\{\leq 1\} \vee X_{3}\{\leq 1\} X_{4}\{\leq 1\}\right)\end{array}$ \\
\hline & $\begin{array}{l}S\{\geq 1\} \\
=X_{1}\{\geq 1\} \vee X_{2}\{\geq 1\} \\
\vee X_{3}\{\geq 1\} X_{4}\{\geq 1\}\end{array}$ & $\begin{array}{c}S\{\leq 0\}=X_{1}\{\leq 0\} X_{2}\{\leq 0\}\left(X_{3}\{\leq 0\}\right. \\
\left.\vee X_{4}\{\leq 0\}\right)\end{array}$ \\
\hline \multirow{3}{*}{ PRE } & $\begin{array}{l}S\{\geq 3\} \\
=X_{1}\{\geq 3\} \\
\vee X_{1}\{<3\} X_{2}\{\geq 3\} X_{3}\{\geq 3\} X_{4}\{\geq 3\},(14)\end{array}$ & $\begin{aligned} S\{\leq 2\}=X_{1}\{\leq 2\} & \left(X_{2}\{\leq 2\}\right. \\
& \vee X_{2}\{>2\}\left(X_{3}\{\leq 2\}\right. \\
& \left.\left.\vee X_{3}\{>2\} X_{4}\{\leq 2\}\right)\right)\end{aligned}$ \\
\hline & $\begin{array}{l}S\{\geq 2\} \\
=X_{1}\{\geq 2\} \vee X_{1}\{<2\} X_{2}\{\geq 2\}\left(\left(X_{3}\{\geq 2\}\right.\right. \\
\left.\vee X_{3}\{<2\} X_{4}\{\geq 2\}\right)\end{array}$ & $\begin{array}{l}S\{\leq 1\} \\
=X_{1}\{\geq 1\}\left(\left(X_{2}\{\leq 1\} \vee X_{2}\{>1\} X_{3}\{\leq 1\} X_{4}\{\leq 1\}\right),\right.\end{array}$ \\
\hline & $\begin{array}{l}S\{\geq 1\} \\
=X_{1}\{\geq 1\} \vee X_{1}\{<1\}\left(X_{2}\{\geq 1\}\right. \\
\left.\vee X_{1}\{<1\} X_{2}\{\geq 1\} X_{4}\{\geq 1\}\right)\end{array}$ & $\begin{array}{l}S\{\leq 0\} \\
=X_{1}\{\leq 0\} X_{2}\{\leq 0\}\left(X_{3}\{\leq 0\}\right. \\
\left.\vee X_{3}\{>0\} X_{4}\{\leq 0\}\right)\end{array}$ \\
\hline
\end{tabular}

The lower half of Table 1 replicates the upper half, using probability-ready expressions (PREs) (Rushdi, 1987, 1988, 2010; Rushdi and Rushdi, 2017; Rushdi, 2019). These PREs can be readily converted (on a one-to-one basis) into expected values by replacing the logical ORing and ANDing by arithmetic counterparts of addition and multiplication and replacing component instances by their expected values (see Sec. 6 for details).

\section{Characterization via Various Karnaugh Maps}

\subsection{Characterization via a Multi-Valued Karnaugh map}

The system under study can be characterized through the exhaustive listing of all the entries of its function table, conveniently constructed as a multi-valued Karnaugh map (MVKM). This map, shown in Figure 1, has four four-valued inputs $X_{1}, X_{2}, X_{3}$ and $X_{4}$, each belonging to $\{0,1,2,3\}$, and four-valued entries, also belonging to $\{0,1,2,3\}$. This map is considerably large as it has $4^{4}=256$ cells, and is an adaptation of a map of eight binary variables that has the same number of cells $\left(2^{8}\right.$ =256). A variant of Figure 1 (not shown herein due to space limitations) would have been a set of three maps, again with four four-valued inputs $X_{1}, X_{2}, X_{3}$ and $X_{4}$ each, and with binary outputs representing either the instances $\{S\{1\}, S\{2\}, S\{3\}\}$, or the successes $\{S\{\geq 1\}, S\{\geq 2\}, S\{\geq 3\}\}$ (see, e.g., Rushdi and Alsayegh, 2019). Though the MVKM of Figure 1 serves as a natural, unique, and complete representation of the present coherent multi-state system, it has many 
International Journal of Mathematical, Engineering and Management Sciences

Vol. 6, No. 1, 309-321, 2021

https://doi.org/10.33889/IJMEMS.2021.6.1.020

superfluous entries. In fact, listing of all 256 map entries is unnecessary, as it suffices to indicate the entries of the minimal upper vectors (MUVs) (shown red in Figure 1), or, alternatively, to highlight the entries of the maximal lower vectors (MLVs) (shown green in Figure 1) (Lisnianski and Levitin, 2003). The MVKM in Figure 1 can be used to visualize each of the properties of causality, monotonicity, and relevancy, which when added together amount to labelling the present multi-state system as a coherent one (Rushdi and Al-Amoudi, 2019).

\begin{tabular}{|c|c|c|c|c|c|c|c|c|c|c|c|c|c|c|c|c|c|c|}
\hline \multirow{2}{*}{$\begin{array}{l}X_{1} \\
X_{2}\end{array}$} & \multicolumn{4}{|c|}{0} & \multicolumn{4}{|c|}{1} & \multicolumn{4}{|c|}{2} & \multicolumn{4}{|c|}{3} & & \\
\hline & 0 & 1 & 2 & 3 & 0 & 1 & 2 & 3 & 0 & 1 & 2 & 3 & 0 & 1 & 2 & 3 & & \\
\hline & 0 & 1 & 1 & 1 & 1 & 1 & 1 & 1 & 2 & 2 & 2 & 2 & 3 & 3 & 3 & 3 & 0 & \multirow{4}{*}{0} \\
\hline & 0 & 1 & 1 & 1 & 1 & 1 & 1 & 1 & 2 & 2 & 2 & 2 & 3 & 3 & 3 & 3 & 1 & \\
\hline & 0 & 1 & 2 & 2 & 1 & 1 & 2 & 2 & 2 & 2 & 2 & 2 & 3 & 3 & 3 & 3 & 2 & \\
\hline & 0 & 1 & 2 & 2 & 1 & 1 & 2 & 2 & 2 & 2 & 2 & 2 & 3 & 3 & 3 & 3 & 3 & \\
\hline & 0 & 1 & 1 & 1 & 1 & 1 & 1 & 1 & 2 & 2 & 2 & 2 & 3 & 3 & 3 & 3 & 0 & \multirow{4}{*}{1} \\
\hline & 1 & 1 & 1 & 1 & 1 & 1 & 1 & 1 & 2 & 2 & 2 & 2 & 3 & 3 & 3 & 3 & 1 & \\
\hline & 1 & 1 & 2 & 2 & 1 & 1 & 2 & 2 & 2 & 2 & 2 & 2 & 3 & 3 & 3 & 3 & 2 & \\
\hline & 1 & 1 & 2 & 2 & 1 & 1 & 2 & 2 & 2 & 2 & 2 & 2 & 3 & 3 & 3 & 3 & 3 & \\
\hline & 0 & 1 & 2 & 2 & 1 & 1 & 2 & 2 & 2 & 2 & 2 & 2 & 3 & 3 & 3 & 3 & 0 & \multirow{4}{*}{2} \\
\hline & 1 & 1 & 2 & 2 & 1 & 1 & 2 & 2 & 2 & 2 & 2 & 2 & 3 & 3 & 3 & 3 & 1 & \\
\hline & 1 & 1 & 2 & 2 & 1 & 1 & 2 & 2 & 2 & 2 & 2 & 2 & 3 & 3 & 3 & 3 & 2 & \\
\hline & 1 & 1 & 2 & 2 & 1 & 1 & 2 & 2 & 2 & 2 & 2 & 2 & 3 & 3 & 3 & 3 & 3 & \\
\hline & 0 & 1 & 2 & 2 & 1 & 1 & 2 & 2 & 2 & 2 & 2 & 2 & 3 & 3 & 3 & 3 & 0 & \multirow{4}{*}{3} \\
\hline & 1 & 1 & 2 & 2 & 1 & 1 & 2 & 2 & 2 & 2 & 2 & 2 & 3 & 3 & 3 & 3 & 1 & \\
\hline & 1 & 1 & 2 & 2 & 1 & 1 & 2 & 2 & 2 & 2 & 2 & 2 & 3 & 3 & 3 & 3 & 2 & \\
\hline & 1 & 1 & 2 & 3 & 1 & 1 & 2 & 3 & 2 & 2 & 2 & 3 & 3 & 3 & 3 & 3 & 3 & \\
\hline & & & & & & & & & & & & & & & & & $X_{4}$ & $X_{3}$ \\
\hline
\end{tabular}

Figure 1. A multi-valued Karnaugh map (MVKM) for the structure function of a multi-state coherent threshold system. Map entries are superfluous, since it suffices to state the minimal upper vectors (cells with entries colored in red) or the maximal lower vectors (cells with entries colored in green).

The MVKM in Figure 1 is obtained from the algebraic equations (8)-(10) (or from equations (14)-(16)) as follows. The prime implicants (PIs) of $S\{\geq 3\}$ in (8) (or its disjoint implicants (DIs) in (14)) are drawn, and each of the entries of their cells is taken as 3. Then, the PIs of $S\{\geq 2\}$ in (9) (or its DIs in (15)) are constructed, and each of their cells is entered with 2 unless it is already entered with 3. Finally, the PIs of $S\{\geq 1\}$ in (10) (or its DIs in (16)) are used to cover the cells to be entered with 1 unless they are already entered with 2 or 3 . Any cell that is so far un-entered is entered with 0 (or left blank). Figure 2 explains and demonstrates the key step in the above procedure by highlighting (in light green) the PI loop $X_{2}\{\geq 2\} X_{4}\{\geq 2\}$ of $S\{\geq 2\}$. Note that the highest cell of this loop is the all-3 cell $X_{1}\{3\} X_{2}\{3\} X_{3}\{3\} X_{4}\{3\}$, while its lowest cell is the cell $X_{1}\{0\} X_{2}\{2\} X_{3}\{0\} X_{4}\{2\}$, which is a minimal upper vector (MUV). Figure 2 demonstrates clearly that a PI of success at level $j$ should not be confused with (albeit profoundly related to) the MUV that serves as its extremely lowest cell. This PI loop consists of cells extending (inclusively) between the MUV cell and the all- $m$ cell. 
International Journal of Mathematical, Engineering and Management Sciences

Vol. 6, No. 1, 309-321, 2021

https://doi.org/10.33889/IJMEMS.2021.6.1.020

\begin{tabular}{|c|c|c|c|c|c|c|c|c|c|c|c|c|c|c|c|c|c|c|}
\hline$X_{1}$ & \multicolumn{4}{|c|}{0} & \multicolumn{4}{|c|}{1} & \multicolumn{4}{|c|}{2} & \multicolumn{4}{|c|}{3} & & \\
\hline$X_{2}$ & 0 & 1 & 2 & 3 & 0 & 1 & 2 & 3 & 0 & 1 & 2 & 3 & 0 & 1 & 2 & 3 & & \\
\hline & 0 & 1 & 1 & 1 & 1 & 1 & 1 & 1 & 2 & 2 & 2 & 2 & 3 & 3 & 3 & 3 & 0 & \multirow{4}{*}{0} \\
\hline & 0 & 1 & 1 & 1 & 1 & 1 & 1 & 1 & 2 & 2 & 2 & 2 & 3 & 3 & 3 & 3 & 1 & \\
\hline & 0 & 1 & 2 & 2 & 1 & 1 & 2 & 2 & 2 & 2 & 2 & 2 & 3 & 3 & 3 & 3 & 2 & \\
\hline & 0 & 1 & 2 & 2 & 1 & 1 & 2 & 2 & 2 & 2 & 2 & 2 & 3 & 3 & 3 & 3 & 3 & \\
\hline & 0 & 1 & 1 & 1 & 1 & 1 & 1 & 1 & 2 & 2 & 2 & 2 & 3 & 3 & 3 & 3 & 0 & \multirow{4}{*}{1} \\
\hline & 1 & 1 & 1 & 1 & 1 & 1 & 1 & 1 & 2 & 2 & 2 & 2 & 3 & 3 & 3 & 3 & 1 & \\
\hline & 1 & 1 & 2 & 2 & 1 & 1 & 2 & 2 & 2 & 2 & 2 & 2 & 3 & 3 & 3 & 3 & 2 & \\
\hline & 1 & 1 & 2 & 2 & 1 & 1 & 2 & 2 & 2 & 2 & 2 & 2 & 3 & 3 & 3 & 3 & 3 & \\
\hline & 0 & 1 & 2 & 2 & 1 & 1 & 2 & 2 & 2 & 2 & 2 & 2 & 3 & 3 & 3 & 3 & 0 & \multirow{4}{*}{2} \\
\hline & 1 & 1 & 2 & 2 & 1 & 1 & 2 & 2 & 2 & 2 & 2 & 2 & 3 & 3 & 3 & 3 & 1 & \\
\hline & 1 & 1 & 2 & 2 & 1 & 1 & 2 & 2 & 2 & 2 & 2 & 2 & 3 & 3 & 3 & 3 & 2 & \\
\hline & 1 & 1 & 2 & 2 & 1 & 1 & 2 & 2 & 2 & 2 & 2 & 2 & 3 & 3 & 3 & 3 & 3 & \\
\hline & 0 & 1 & 2 & 2 & 1 & 1 & 2 & 2 & 2 & 2 & 2 & 2 & 3 & 3 & 3 & 3 & 0 & \multirow{4}{*}{3} \\
\hline & 1 & 1 & 2 & 2 & 1 & 1 & 2 & 2 & 2 & 2 & 2 & 2 & 3 & 3 & 3 & 3 & 1 & \\
\hline & 1 & 1 & 2 & 2 & 1 & 1 & 2 & 2 & 2 & 2 & 2 & 2 & 3 & 3 & 3 & 3 & 2 & \\
\hline & 1 & 1 & 2 & 3 & 1 & 1 & 2 & 3 & 2 & 2 & 2 & 3 & 3 & 3 & 3 & 3 & 3 & \\
\hline & & & & & & & & & & & & & & & & & $X_{4}$ & $X_{3}$ \\
\hline
\end{tabular}

Figure 2. The MVKM of Figure 1 redrawn to highlight the relation between a prime implicant of success (failure) at level 2 and its characterizing minimal upper vector (maximal lower vectors).

In a 'dual' fashion, the MVKM might be obtained from equations (11)-(13) (or from equations (17)-(20)) as follows. The PIs of $S\{\leq 0\}$ in (13) (or its DIs in (19)) are drawn, and each of the entries of their cells is taken as 0 . Then, the PIs of $S\{\leq 1\}$ in (12) (or its DIs in (18)) are constructed, and each of their cells is entered with 1 unless it is already entered with 0 . Finally, the PI's of $S\{\leq 2\}$ in (11) (or its DIs in (17)) are used to cover the cells to be entered with 2 unless they are already entered with 0 or 1 . Any cell that is still blank is left so or filled in with 3 . Figure 2 again demonstrates the key step in this dual procedure by highlighting (in yellow) the PI loop $X_{1}\{\leq 1\} X_{3}\{\leq 1\} X_{4}\{\leq 1\}$ of $S\{\leq 1\}$. Note that the lowest cell of this loop is the all-0 cell $X_{1}\{0\} X_{2}\{0\} X_{3}\{0\} X_{4}\{0\}$, while its highest cell is the cell $X_{1}\{1\} X_{2}\{3\} X_{3}\{1\} X_{4}\{1\}$, which is a maximal lower vector (MLV). Figure 2 again demonstrates clearly that a PI of failure at level $j$ should not be literally identified with its defining MLV that serves as its extremely highest cell. This PI loop comprises all cells extending (inclusively) between the all-0 cell and the MLV cell.

\subsection{Characterization via $\boldsymbol{m}$ Conventional Karnaugh maps}

This subsection is of crucial importance, since it clearly conveys the essence of the paradigm of handling binary-imaged multi-state systems via pure switching-algebraic tools. We replace the MVKM of Figure 1 by a set of $m$ (here 3) conventional Karnaugh maps (CKMs) of appropriate input variables. A map in this set represents $S\{\geq j\}$ as a function of $\boldsymbol{X}\{\geq j\}$ for $(j=3,2$, and 1$)$. It might also be viewed to represent the complementing entity $S\{<j\}$ as a function of $\boldsymbol{X}\{<$ $j$ \} again for $(j=3,2$, and 1 ), or, equivalently $S\{\leq j\}$ as a function of $\boldsymbol{X}\{\leq j\}$ (for $j=$ 2,1 , and 0 ). The top part of Figure 3 constitutes such a set of three maps covered by minimal PI loops (representing (8)-(10) for true (upper) vectors and (11)-(13) for false (lower) vectors), while 
International Journal of Mathematical, Engineering and Management Sciences

Vol. 6, No. 1, 309-321, 2021

https://doi.org/10.33889/IJMEMS.2021.6.1.020

the bottom part of Figure 3 comprises an equivalent set covered by disjoint loops (representing (14)-(16) for true vectors and (17)-(19) for false vectors). Each of the upper loops in the former set of maps passes through the all-1 cell and has a starred cell whose product might be minimized to yield a minimal upper vector (MUV) (Table 2). Likewise, each of the lower loops therein passes through the all-0 cell and has a double-starred cell whose product might be maximized to yield a maximal lower vector (MLV) (Table 2).

Table 2. Using minimality of an extreme (starred) cell of a prime implicant of success at level $j$ to produce a minimal upper vector, and using maximality of an extreme (double-starred) cell of a prime implicant of failure at level $j$ to produce a maximal lower vector. Since the system is binary-imaged, elements of the MUVs set $\theta(j)$ belong to $\{0, j\}$, while elements of the MLVs set $\sigma(j)$ belong to $\{j, m\}$.

\begin{tabular}{|c|c|c|c|c|}
\hline $\begin{array}{c}\text { Level } \\
j\end{array}$ & Starred Cell & $\begin{array}{c}\text { Minimal } \\
\text { Upper } \\
\text { Vectors } \\
\theta(j)\end{array}$ & Double Starred Cell & $\begin{array}{c}\text { Maximal } \\
\text { Lower } \\
\text { Vectors } \\
\sigma(j-1)\end{array}$ \\
\hline \multirow{2}{*}{3} & $X_{1}\{\geq 3\} X_{2}\{<3\} X_{3}\{<3\} X_{4}\{<3\}$ & $(3,0,0,0)$ & $X_{1}\{\leq 2\}\left(X_{2}\{\leq 2\} X_{3}\{>2\} X_{4}\{>2\}\right.$ & $(2,2,3,3)$ \\
& $X_{1}\{<3\} X_{2}\{\geq 3\} X_{3}\{\geq 3\} X_{4}\{\geq 3\}$ & $(0,3,3,3)$ & $X_{1}\{\leq 2\} X_{2}\{>2\} X_{3}\{\leq 2\} X_{4}\{>2\}$ & $(2,3,2,3)$ \\
& $X_{1}\{\leq 2\} X_{2}\{>2\} X_{3}\{>2\} X_{4}\{\leq 2\}$ & $(2,3,3,2)$ \\
\hline \multirow{2}{*}{2} & $X_{1}\{\geq 2\} X_{2}\{<2\} X_{3}\{<2\} X_{4}\{<2\}$ & $(2,0,0,0)$ & $X_{1}\{\leq 1\}\left(X_{2}\{\leq 1\} X_{3}\{>1\} X_{4}\{>1\}\right.$ & $(1,1,3,3)$ \\
& $X_{1}\{<2\} X_{2}\{\geq 2\} X_{3}\{\geq 2\} X_{4}\{<2\}$ & $(0,2,2,0)$ & $X_{1}\{\leq 1\} X_{2}\{>1\} X_{3}\{\leq 1\} X_{4}\{\leq 1\}$ & $(1,3,1,1)$ \\
\hline \multirow{3}{*}{1} & $X_{1}\{<2\} X_{2}\{\geq 2\} X_{3}\{<2\} X_{4}\{\geq 2\}$ & $(0,2,0,2)$ & & \\
& $X_{1}\{\geq 1\} X_{2}\{<1\} X_{3}\{<1\} X_{4}\{<1\}$ & $(1,0,0,0)$ & $X_{1}\{\leq 0\} X_{2}\{\leq 0\}\left(X_{3}\{\leq 0\} X_{4}\{>0\}\right.$ & $(0,0,0,3)$ \\
& $X_{1}\{<1\}\left(X_{2}\{\geq 1\} X_{3}\{<1\} X_{4}\{<1\}\right.$ & $(0,1,0,0)$ & \\
& $X_{1}\{<1\} X_{2}\{<1\} X_{3}\{\geq 1\} X_{4}\{\geq 1\}$ & $(0,0,1,1)$ & $X_{1}\{\leq 0\} X_{2}\{\leq 0\} X_{3}\{>0\} X_{4}\{\leq 0\}$ & $(0,0,3,0)$ \\
\hline
\end{tabular}

To close this section, we elaborate on the relation between the MVKM of Figure 1 and the binary maps in Figure 3. Figure 4 shows how the aforementioned MVKM can be reduced to represent each of the three successes $S\{\geq 3\}, S\{\geq 2\}$ and $S\{\geq 1\}$, where we have opted herein to produce $S\{\geq 2\}$ only as an illustrative example. The inputs of the resulting map cease to be multivalued but assume the binary values

$X_{i}\{\geq 2\}=X_{i}\{2,3\}=X_{i}\{2\} \vee X_{i}\{3\}$

and

$$
X_{i}\{<2\}=X_{i}\{\leq 1\}=X_{i}\{0,1\}=X_{i}\{0\} \vee X_{i}\{1\}
$$

A single cell in the equivalent map of $X_{i}\{\geq 2\}$ in Figure 3 results from the amalgamation of $2^{4}=$ 16 cells in Figure 1, and it possesses an entry of 1 or 0 when all these 16 cells have entries belonging to $\{2,3\}$ or $\{0,1\}$, respectively. Note that the reduced map in Figure 4 serves only as a partial step towards producing the CKM of $S\{\geq 2\}$ in Figure 3. In fact, Figure 4 is a Marquand diagram (Rushdi, 1997) obtained through combining groups of four cells only in Figure 1. This 
International Journal of Mathematical, Engineering and Management Sciences

Vol. 6, No. 1, 309-321, 2021

https://doi.org/10.33889/IJMEMS.2021.6.1.020

diagram is converted to a CKM by eliminating redundancy (further combining of subgroups of four cells, which must share identical entries) and securing visual adjacency.
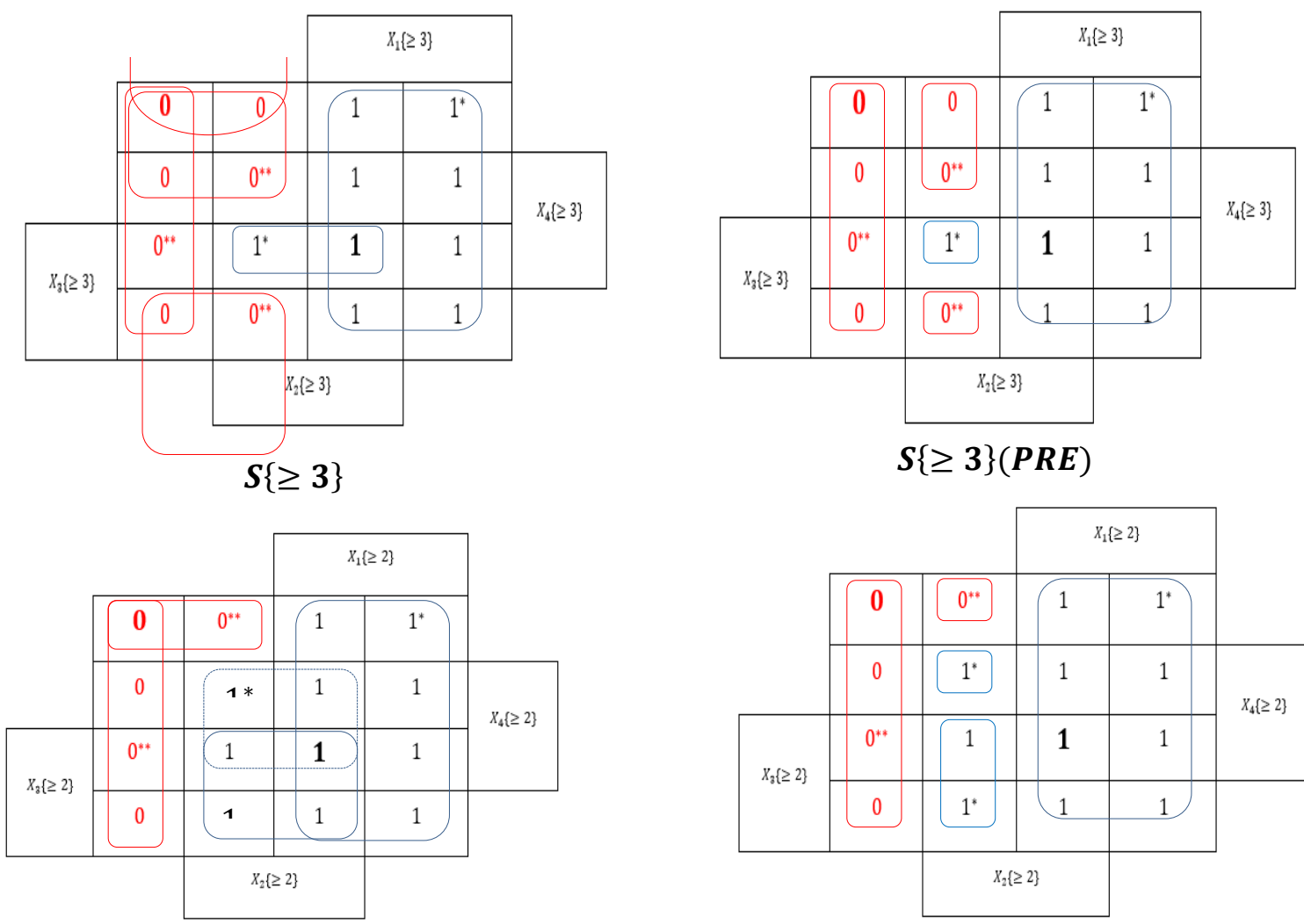

$S\{\geq 2\}$
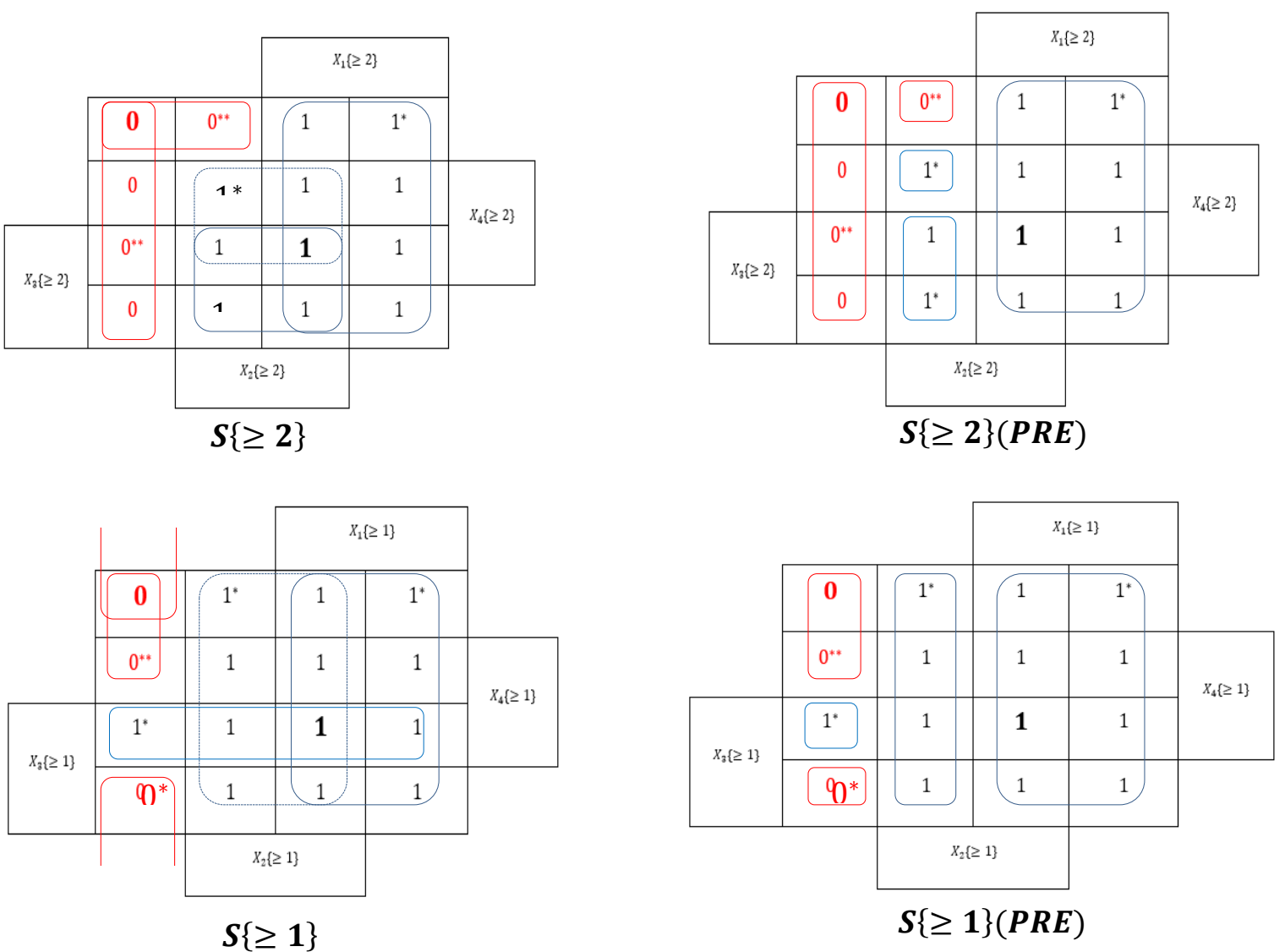

$S\{\geq 1\}$

Figure 3. Prime-implicant (PI) loops for the top six expressions in Table 1, and Disjoint-implicant (DI) loops for the bottom six expressions in Table 1. A starred cell is an enlargement of a minimal upper vector, while a double-starred cell is an enlargement of a maximal lower vector. Each success (1-entered) PI loops passes through the all-1 cell, and each failure (0-entered) PI loop passes through the all- 0 cell. The number of DI loops exactly equals that of the corresponding PI loops, asserting to the shellability of the pertinent expressions. 
International Journal of Mathematical, Engineering and Management Sciences

Vol. 6, No. 1, 309-321, 2021

https://doi.org/10.33889/IJMEMS.2021.6.1.020

\begin{tabular}{|c|c|c|c|c|c|c|c|c|c|c|}
\hline $\mathbf{X}_{1}$ & \multicolumn{4}{|c|}{$<2$} & \multicolumn{4}{|c|}{$\geq 2$} & & \\
\hline $\mathbf{X}_{2}$ & $<2$ & $\geq 2$ & $<2$ & $\geq 2$ & $<2$ & $\geq 2$ & $<2$ & $\geq 2$ & & \\
\hline & 0 & 0 & 0 & 0 & 1 & 1 & 1 & 1 & $<2$ & \multirow{4}{*}{$<2$} \\
\hline & 0 & 1 & 0 & 1 & 1 & 1 & 1 & 1 & $\geq 2$ & \\
\hline & 0 & 0 & 0 & $\mathbf{0}$ & 1 & 1 & 1 & 1 & $<2$ & \\
\hline & 0 & 1 & 0 & 1 & 1 & 1 & 1 & 1 & $\geq 2$ & \\
\hline & 0 & 1 & 0 & 1 & 1 & 1 & 1 & 1 & $<2$ & \multirow{4}{*}{$\geq 2$} \\
\hline & 0 & 1 & 0 & 1 & 1 & 1 & 1 & 1 & $\geq 2$ & \\
\hline & 0 & 1 & 0 & 1 & 1 & 1 & 1 & 1 & $<2$ & \\
\hline & 0 & 1 & 0 & 1 & 1 & 1 & 1 & 1 & $\geq 2$ & \\
\hline & & & & & & & & & $\mathbf{X}_{4}$ & $\mathbf{X}_{3}$ \\
\hline
\end{tabular}

Figure 4. Reduction of the MVKM of Figure 1 to a Marquand diagram, which represents a binary function $S\{\geq 2\}$ in terms of four binary variables. The diagram suffers of redundancy and lack of visual adjacency among neighboring cells, two notorious properties inherited from its parent coherent MVKM, in Figure 1.

\section{Reliability Analysis}

The PRE expressions (14)-(19) might be directly converted (on a one-to-one basis) to the expected values in (22)-(27) (conveniently listed in Table 3) by replacing the AND and OR operators with the multiplication and addition operators and replacing variable instances by their expectations, namely

$$
\begin{aligned}
& E\left\{X_{i}\{j\}\right\}=p_{i j} . \\
& E\left\{X_{i}\{\geq j\}\right\}=p_{i j}+p_{i(j+1)}+\cdots+p_{i m}=1-\left(p_{i 0}+p_{i 1}+\cdots+p_{i(j-1)}\right) .
\end{aligned}
$$

\begin{tabular}{|c|c|c|c|c|}
\hline Level $j$ & \multicolumn{2}{|c|}{ System success at level $j E\{S\{\geq j\}\}$} & \multicolumn{2}{|c|}{$\begin{array}{c}\text { System failure at level } j E\{S\{<j\}\}= \\
E\{S\{\leq(j-1)\}\}\end{array}$} \\
\hline 3 & $p_{13}+\left(1-p_{13}\right) p_{23} p_{33} p_{43}$ & $(22)$ & $\begin{array}{l}\left(1-p_{13}\right)\left(\left(1-p_{23}\right)\right. \\
+p_{23}\left(\left(1-p_{33}\right)+p_{33}\left(1-p_{43}\right)\right)\end{array}$ & $(25)$ \\
\hline 2 & $\begin{array}{c}\left(p_{12}+p_{13}\right)+\left(p_{10}+p_{11}\right) \\
\left(p_{22}+p_{23}\right)\left(\left(p_{32}+p_{33}\right)\right. \\
+\left(p_{30}+p_{31}\right)\left(p_{42}\right. \\
\left.\left.+p_{43}\right)\right)\end{array}$ & $(23)$ & $\begin{aligned}\left(p_{10}+p_{11}\right)\left(\left(p_{20}\right.\right. & \left.+p_{21}\right) \\
& +\left(p_{22}+p_{23}\right)\left(p_{30}\right. \\
& \left.\left.+p_{31}\right)\left(p_{40}+p_{41}\right)\right)\end{aligned}$ & $(26)$ \\
\hline 1 & $\begin{aligned}\left(1-p_{10}\right)+p_{10} & ( \\
+ & \left.1-p_{20}\right) \\
& +p_{20}\left(1-p_{30}\right)(1 \\
& \left.\left.-p_{40}\right)\right)\end{aligned}$ & (24) & $p_{10} p_{20}\left(p_{30}+\left(1-p_{30}\right) p_{40}\right)$ & $(27)$ \\
\hline
\end{tabular}

Table 3. Expectations of system success and failure at each non-zero level $j(j=1,2.3)$. 
International Journal of Mathematical, Engineering and Management Sciences

Vol. 6, No. 1, 309-321, 2021

https://doi.org/10.33889/IJMEMS.2021.6.1.020

Table 4 shows that there are two alternatives for expressing the expectations of various instances of the multivalued system success $S$. Of course, the more compact alternative is preferable.

Table 4. Expectations of each instance of the multivalued system success $S$.

\begin{tabular}{|c|c|c|}
\hline Expectation & Using upper Vectors & Using Lower Vectors \\
\hline$E\{S\{3\}\}$ & $E\{S\{\geq 3\}\}$ & $E\{S\{\leq 3\}\}-E\{S\{\leq 2\}\}$ \\
& & 1 \\
& & \\
\hline$E\{S\{2\}\}$ & $E\{S\{\geq 2\}\}-E\{S\{\geq 3\}\}$ & $E\{S\{\leq 2\}\}-E\{S\{\leq 1\}\}$ \\
\hline$E\{S\{1\}\}$ & $E\{S\{\geq 1\}\}-E\{S\{\geq 2\}\}$ & $E\{S\{\leq 1\}\}-E\{S\{\leq 0\}\}$ \\
\hline$E\{S\{0\}\}$ & $E\{S\{\geq 0\}\}-E\{S\{\geq 1\}\}=1-E\{S\{\geq 1\}\}$ & $E\{S\{\leq 0\}\}$ \\
\hline
\end{tabular}

\section{Conclusions}

This paper dealt with the reliability characterization and analysis of a homogeneous multi-state coherent threshold system of $(m+1)$ states, which is a non-repairable system with independent non-identical components. The paper presents switching-algebraic expressions of both system success and system failure at each non-zero level. These expressions are given as minimal sumof-products formulas or as probability-ready expressions. The paper also suggests several map representations, including a single multi-value Karnaugh map (giving a superfluous representation of the system structure function $S),(m+1)$ maps of binary entries and multivalued inputs representing the instances of $S$, or $m$ maps of binary entries and multi-valued inputs representing the success/failure at every non-zero level of the system. We demonstrated how to reduce these latter maps to conventional Karnaugh maps (CKMs) of much smaller size and of no inherent redundancy. Further system characterizations are also given in terms of minimal upper vectors or maximal lower vectors. Expected values of various system levels are also derived through the immediate transformations of PREs of binary instances of the system multi-valued success/failure. For future work, the present results are to be extended to cover more general multi-state threshold systems that might be noncoherent or lack a well-defined binary image.

\section{Conflict of Interest}

The authors assert that no conflict of interest exists.

\section{Acknowledgement}

The first-named author (AMR) benefited greatly from (and is sincerely grateful for) his earlier collaboration and enlightening discussions with Engineer Mahmoud Ali Rushdi, Munich, Germany. 
International Journal of Mathematical, Engineering and Management Sciences

Vol. 6, No. 1, 309-321, 2021

https://doi.org/10.33889/IJMEMS.2021.6.1.020

\section{References}

Ansell, J.I., \& Bendell, A. (1987). On alternative definitions of multistate coherent systems. Optimization, 18(1), 119-136.

Barlow, R.E., \& Wu, A.S. (1978). Coherent systems with multi-state components. Mathematics of Operations Research, 3(4), 275-281.

Boedigheimer, R.A., \& Kapur, K.C. (1994). Customer-driven reliability models for multistate coherent systems. IEEE Transactions on Reliability, 43(1), 46-50.

Ding, Y., Zio, E., Yanfu, L., Cheng, L., \& Wu, Q. (2012). Definition of multi-state weighted k-out-of-n: F systems. International Journal of Performability Engineering, 8(2), 217-219.

El-Neweihi, E., Proschan, F., \& Sethuraman, J. (1978). Multistate coherent systems. Journal of Applied Probability, 15(4), 675-688.

Eryilmaz, S. (2015). Capacity loss and residual capacity in weighted k-out-of-n: G systems. Reliability Engineering \& System Safety, 136, 140-144.

Eryilmaz, S., \& Bozbulut, A.R. (2019). Reliability analysis of weighted-k-out-of-n system consisting of three-state components. Proceedings of the Institution of Mechanical Engineers, Part O: Journal of Risk and Reliability, 233(6), 972-977.

Griffith, W.S. (1980). Multistate reliability models. Journal of Applied Probability, 17(3), 735-744.

Huang, J., \& Zuo, M.J. (2004). Dominant multi-state systems. IEEE transactions on Reliability, 53(3), 362368.

Hudson, J.C., \& Kapur, K.C. (1983). Reliability analysis for multistate systems with multistate components. AIIE Transactions, 15(2), 127-135.

Janan, X. (1985). On multistate system analysis. IEEE Transactions on Reliability, 34(4), 329-337.

Khorshidi, H.A., Gunawan, I., \& Ibrahim, M.Y. (2015). On reliability evaluation of multistate weighted kout-of-n system using present value. The Engineering Economist, 60(1), 22-39.

Kumar, A., \& Singh, S.B. (2018). Signature reliability of linear multi-state sliding window system. International Journal of Quality \& Reliability Management, 35(10), 2403-2413.

Li, X., You, Y., \& Fang, R. (2016). On weighted k-out-of-n systems with statistically dependent component lifetimes. Probability in the Engineering and Informational Sciences, 30(4), 533-546.

Lisnianski, A., \& Levitin, G. (2003). Multi-state system reliability: assessment, optimization and applications (Vol. 6). World Scientific Publishing Company, Singapore.

Meenkashi, K., Singh, S.B., \& Kumar, A. (2019). Reliability analysis of multi-state complex system with multi-state weighted subsystems. International Journal of Quality \& Reliability Management, 36(4), 552-568.

Mo, Y., Xing, L., Amari, S.V., \& Dugan, J.B. (2015). Efficient analysis of multi-state k-out-of-n systems. Reliability Engineering \& System Safety, 133, 95-105.

Ram, M. (2013). On system reliability approaches: a brief survey, International Journal of System Assurance, Engineering, and Management, 4(2), 101-117.

Rushdi, M.A.M., Ba-Rukab, O.M. \& Rushdi, A.M. (2016). Multidimensional recursive relations and mathematical induction techniques: The case of failure frequency of k-out-of-n systems. Journal of King Abdulaziz University: Engineering Sciences, 27(2), 15-31.

Rushdi, A.M.A. \& Al-Amoudi, M.A. (2019), Reliability analysis of a multi-state system using multi-valued logic. IOSR Journal of Electronics and Communication Engineering, 14(1), 1-10. 
International Journal of Mathematical, Engineering and Management Sciences

Vol. 6, No. 1, 309-321, 2021

https://doi.org/10.33889/IJMEMS.2021.6.1.020

Rushdi, A.M. (1987). A switching-algebraic analysis of consecutive-k-out-of-n: F systems. Microelectronics and Reliability, 27(1), 171-174.

Rushdi, A.M. (1988). A switching-algebraic analysis of circular consecutive-k-out-of-n: F systems. Reliability Engineering \& System Safety, 21(2), 119-127.

Rushdi, A.M. (1990). Threshold systems and their reliability. Microelectronics and Reliability, 30(2), 299312.

Rushdi, A.M. (1997). Karnaugh map, Encyclopedia of Mathematics, Supplement Volume I: pp. 327-328, In: Hazewinkel, M. (ed) Kluwer Academic publishers, Boston, USA.

Rushdi, A.M. (2010). Partially-redundant systems: Examples, reliability, and life expectancy. International Magazine on Advances in Computer Science and Telecommunications, 1(1), 1-13.

Rushdi, A.M., \& Abdulghani, A.A. (1993). A comparison between reliability analyses based primarily on disjointness or statistical independence: The case of the generalized INDRA network. Microelectronics and Reliability, 33(7), 965-978.

Rushdi, A.M., \& Alturki, A.M. (2018). Novel representations for a coherent threshold reliability system: a tale of eight signal flow graphs. Turkish Journal of Electrical Engineering \& Computer Sciences, 26(1), 257-269.

Rushdi, A.M., \& Goda, A.S. (1985). Symbolic reliability analysis via Shannon's expansion and statistical independence. Microelectronics and Reliability, 25(6), 1041-1053.

Rushdi, A.M., \& Rushdi, M.A. (2017). Switching-algebraic analysis of system reliability. In: Ram, M. \& Davim, J.P. (eds) Advances in Reliability and System Engineering. Springer, Cham, pp. 139-161.

Rushdi, A.M.A. (2018). Utilization of Karnaugh maps in multi-value qualitative comparative analysis. International Journal of Mathematical, Engineering and Management Sciences, 3(1), 28-46.

Rushdi, A.M.A. (2019). Utilization of symmetric switching functions in the symbolic reliability analysis of multi-state k-out-of-n systems. International Journal of Mathematical, Engineering and Management Science, 4(2), 306-326.

Rushdi, A.M.A., \& Al-Amoudi, M.A. (2018). Switching-algebraic analysis of multi-state system reliability. Journal of Engineering Research and Reports, 3(3), 1-22.

Rushdi, A.M.A., \& Alsayegh, A.B. (2019). Reliability analysis of a commodity-supply multi-state system using the map method. Journal of Advances in Mathematics and Computer Science, 31(2), 1-17.

Rushdi, A.M.A., \& Alturki, A.M. (2015). Reliability of coherent threshold systems. Journal of Applied Sciences, 15(3), 431-443.

Rushdi, A.M.A., \& Bjaili, H.A. (2016). An ROBDD algorithm for the reliability of double-threshold systems. British Journal of Mathematics and Computer Science, 19(6), 1-17.

Rushdi, A.M.A., \& Hassan, A.K. (2015). Reliability of migration between habitat patches with heterogeneous ecological corridors. Ecological Modelling, 304, 1-10.

Rushdi, A.M.A., \& Hassan, A.K. (2016). An exposition of system reliability analysis with an ecological perspective. Ecological Indicators, 63, 282-295.

Rushdi, R.A., \& Rushdi, A.M. (2018). Karnaugh-map utility in medical studies: the case of fetal malnutrition. International Journal of Mathematical, Engineering and Management Sciences, 3(3), 220-244.

Salehi, M., Shishebor, Z., \& Asadi, M. (2019). On the reliability modeling of weighted k-out-of-n systems with randomly chosen components. Metrika, 82(5), 589-605. 
International Journal of Mathematical, Engineering and Management Sciences

Vol. 6, No. 1, 309-321, 2021

https://doi.org/10.33889/IJMEMS.2021.6.1.020

Tian, Z., Zuo, M.J., \& Yam, R.C. (2008). Multi-state k-out-of-n systems and their performance evaluation. IIE Transactions, 41(1), 32-44.

Wood, A.P. (1985). Multistate block diagrams and fault trees. IEEE Transactions on Reliability, 34(3), 236-240.

Zuo, M.J., Huang, J., \& Kuo, W. (2003). Multi-state k-out-of-n systems. In Handbook of Reliability Engineering (pp. 3-17). Springer, London. 